\title{
Etude des particularités de la poule Fayoumi II. - Résistance à la coccidiose (Eimeria tenella) des poussins Fayoumi, Rhode-Island et de leur croisement
}

\author{
N. HAMET et P. MÉRAT * \\ avec la collaboration technique de Francine Bertrand et G. Coquerelle \\ Ministère de l'Agriculture, Direction de la Qualité, Institut d'Elevage, \\ de Pathologie et d'Hygiène alimentaire, Laboratoire de Parasitologie, F 22440 Ploufragan \\ * I.N.R.A., Laboratoire de Génétique factorielle, Centre de Recherches zootechniques, \\ F 78350 Jouy-en-Josas
}

\begin{abstract}
Résumé
Des poussins des races Fayoumi, Rhode-Island et des deux croisements réciproques entre ces races, ont été répartis entre un lot "témoin" non contaminé et un lot "inoculé " dont chaque individu recevait 200000 ookystes (Eimeria tenella) à 15 jours d'âge. Tous les poussins étaient abattus à 23 jours. La mortalité entre l'inoculation et l'abattage, le gain de poids des survivants, l'importance des lésions caecales appréciée par une note de 0 à 4 , étaient enregistrés individuellement. La consommation d'aliment et d'eau était notée par groupes. Enfin, l'excrétion journalière d'ookystes et l'aspect des matières fécales étaient relevés sur un échantillon de chaque race ou croisement. Pour tous ces critères, les poussins Fayoumi apparaissent les plus résistants à Eimeria tenella. En particulier, leur mortalité est nulle, leur gain de poids n'est abaissé que de $4 \mathrm{p}$. cent par comparaison au lot témoin, et leurs lésions caecales sont dans l'ensemble moins importantes. A tous ces points de vue, les poussins Rhode-Island sont les plus sensibles en moyenne, et les deux croisements sont intermédiaires.
\end{abstract}

\section{Introduction}

La race de poules Fayoumi, répandue en Egypte, a la réputation d'être relativement résistante à divers agents pathogènes. Prince (1958) met en évidence une résistance d'embryons Fayoumi à une inoculation de virus du Sarcome de Rous. Selon Nordskog \& Philips (1960), une lignée de cette race a été trouvée plus résistante à la maladie de Marek que des lignées de Leghorn et de races lourdes et leurs croisements. Khodali \& Shomein (1974) trouvent la Fayoumi et d'autres races locales au Soudan plus résistantes que la Leghorn à la spirochétose. C'est également une opinion répandue en Egypte que la Fayoumi est particulièrement résistante à une infection par coccidiose, une sélection naturelle s'étant vraisemblablement exercée dans les conditions souvent difficiles où elle est élevée. Nous avons voulu vérifier plus précisément ce dernier point, par inoculation contrôlée de l'espèce Eimeria tenella à des poussins Fayoumi, des poussins de race Rhode-Island et des poussins provenant des croisements réciproques entre ces deux races. 


\section{Matériel et méthodes}

\section{A. Matériel animal - Types génétiques comparés}

La lignée Fayoumi entretenue au Laboratoire de Génétique factorielle, à Jouyen-Josas, était issue d'œufs fécondés provenant de reproducteurs d'une lignée sélectionnée sur le nombre d'cufs à l'Université du Caire (Department of Animal Breeding) en décembre 1978.

Le 29 avril 1980, une éclosion était issue de 16 parquets pedigree, 8 avec un coq Fayoumi, 8 avec un coq Rhode-Island (lignée M 99).

A chaque coq étaient appariées 4 poules Fayoumi et 4 poules Rhode-Island. La descendance comprenait ainsi 4 types génétiques, les 2 lignées parentales et leurs deux croisements réciproques de $1^{\text {re }}$ génération. Les poussins mâles étaient envoyés à la Station Expérimentale d'Aviculture à Ploufragan. Les nombres d'animaux gardés après histogramme réalisé à l'âge de 13 jours, étaient les suivants :

$\begin{array}{lr}\text { - R.I.R. } & : 38 \\ \text { - Fayoumi } & : 76 \\ \text { - } \sigma \text { R.I.R. } \times \text { q Fayoumi }: 52 \\ \text { - } \sigma \text { Fayoumi } \times \text { q R.I.R. : } 64\end{array}$

\section{B. Lots, conditions expérimentales, inoculation}

Les poussins de chaque type génétique étaient répartis en deux lots, témoin (T) et contaminé $(C)$. Ils étaient placés dans des cages de 4 animaux chacune. L'aliment distribué, de type "poulet de chair $1^{\mathrm{er}}$ âge ", contenait $20 \mathrm{p}$. cent de protéines totales et $2850 \mathrm{Kcal} / \mathrm{kg}$ d'énergie métabolisable. L'âge des animaux était de 13 jours en début d'expérience et de 23 jours à l'abattage.

L'inoculation de l'agent infectieux était faite à 15 jours d'âge. La dose inoculée était de 200000 ookystes sporulés de la souche $\mathrm{PT}_{5}$ d'Eimeria tenella remultipliée au Laboratoire de Parasitologie de la Station depuis 1965.

\section{Critères observés ou mesurés}

Les critères suivants sont enregistrés individuellement :

- gain de poids de 13 jours d'âge (soit 2 jours avant l'inoculation) à 23 jours (soit 8 jours après l'inoculation). Ce gain a été également noté de 13 jours d'âge à l'âge de la mort si celle-ci est survenue avant 23 jours ;

- mortalité liée à la coccidiose de l'âge de 20 jours (soit 5 jours après le début de la maladie) à l'abattage ;

- gravité des lésions caecales sur les survivants à l'abattage, notée selon l'échelle de Johnson \& ReID (1970) de 0 (pas de lésions macroscopiques) à 4 (maximum des lésions ou mort de coccidiose). 
Les mesures ci-après étaient faites par groupes de 4 poussins élevés dans la même cage :

- excrétion ookystale journalière par animal, du $5^{\star}$ au $7^{\mathrm{e}}$ jour inclus après inoculation (sur une cage par type génétique); la méthode d'évaluation du nombre d'ookystes est celle décrite par HAMET et al. (1981) ;

- aspect des matières fécales noté quotidiennement du $5^{\mathrm{e}}$ au $7^{\mathrm{e}}$ jour après l'inoculation, selon l'échelle suivante :

$0=$ matières fécales normales

$1=$ traces d'hémorragies

$2=$ quelques taches de sang

$3=$ nombreuses taches de sang

$4=$ matières fécales très hémorragiques ;

- consommations moyennes journalières d'eau et d'aliment par individu et indice de consommation, de 13 à 23 jours d'âge (sur l'ensemble des poussins).

\section{Analyses statistiques}

Les valeurs moyennes par type génétique sont données pour toutes les variables, mais seules les observations ou mesures faites individuellement font l'objet d'une analyse statistique. Pour le gain de poids des survivants, une analyse de variance avec les facteurs "type génétique " (4 niveaux) et "traitement " $(2$ niveaux $)$ a permis de tester, outre les effets de ces facteurs, leur interaction. Une analyse similaire a été faite sur les poids en fin d'expérience, et d'autre part sur le rapport individuel du gain de poids au poids corporel initial. La mortalité des quatre types génétiques a été comparée par un test de $\chi^{2}$. Il en est de même des notes de lésions caecales. Pour ces dernières, la note 2 était la plus fréquente et la note 4 était rare. En conséquence, nous avons comparé pour les 4 génotypes, la fréquence de deux classes, l'une regroupant les notes 1 et 2 , l'autre les notes 3 et 4 .

Enfin, intra-type génétique, le gain de poids a été comparé pour les différentes notes de lésions (analyse de variancè) en vue d'évaluer une liaison possible entre ces deux variables.

\section{Résultats}

Le tableau 1 donne les valeurs moyennes, par type génétique, pour toutes les variables mentionnées ci-dessus. La mortalité n'est indiquée que pour le lot contaminé ; elle est nulle dans le lot témoin.

Le tableau 2 contient les analyses de variance pour les caractères " gain de poids », "poids à l'abattage » et « rapport du gain de poids au poids initial » pour les survivants à 23 jours, avec le test des effets "type génétique », «traitement » et de leur interaction. 


\section{TABLEAU 1}

Valeur moyenne par caractère pour les deux lignées et leurs croisements.

Mean value for each trait for strains and crosses.

\begin{tabular}{|c|c|c|c|c|c|}
\hline \multirow{2}{*}{\multicolumn{2}{|c|}{$\begin{array}{l}\text { Caractère et lot } \\
(\mathrm{T}=\text { témoin } \\
\mathrm{C}=\text { contaminé) }\end{array}$}} & \multicolumn{4}{|c|}{ Lignée ou croisement } \\
\hline & & \multirow{3}{*}{$\begin{array}{c}\begin{array}{c}\text { R.I.R. } \\
\text { (lignée M99) }\end{array} \\
\begin{array}{c}19 \\
19\end{array}\end{array}$} & \multirow{3}{*}{$\begin{array}{c}\text { Fayoumi } \\
38 \\
38\end{array}$} & \multirow{3}{*}{$\begin{array}{c}\text { R.I.R.X. } \\
\text { Fayoumi }\end{array}$} & \multirow{3}{*}{$\begin{array}{c}\text { Fayoumi } \\
\times \text { R.I.R. } \\
32 \\
32\end{array}$} \\
\hline Effectifs de départ & (T) & & & & \\
\hline & (C) & & & & \\
\hline \multirow[t]{2}{*}{ Poids à $13 \mathrm{j}(\mathrm{g})$} & (T) & 126,5 & 93,6 & 112,5 & 129,4 \\
\hline & (C) & 128,5 & 95,7 & 113,7 & 128,2 \\
\hline \multirow{2}{*}{$\begin{array}{l}\text { Poids à } 23 \mathrm{j} \text { (g) } \\
\text { (survivants) }\end{array}$} & (T) & 260,9 & 182,7 & 225,2 & 255,6 \\
\hline & (C) & 228,0 & 180,9 & 201,5 & 228,8 \\
\hline \multirow{2}{*}{$\begin{array}{l}\text { Gain de poids absolu } \\
\text { de } 13 \text { à } 23 \mathrm{j} \text { (g) (survivants) }\end{array}$} & (T) & 134,4 & 89,1 & 112,9 & 126,2 \\
\hline & (C) & 100,4 & 85,2 & 87,9 & 102,3 \\
\hline \multicolumn{2}{|c|}{ Différence $(C)-(T)$ en $p$. cent de $T$} & 25,3 & 4,4 & 22,1 & 18,9 \\
\hline \multirow{2}{*}{$\begin{array}{l}\text { Gain de poids relatif rapporté } \\
\text { au poids initial (survivants) }\end{array}$} & (T) & 0,51 & 0,48 & 0,49 & 0,49 \\
\hline & (C) & 0,40 & 0,47 & 0,41 & 0,42 \\
\hline Mortalité (p. cent) & (C) & 31,6 & 0,0 & 11,5 & 9,7 \\
\hline $\begin{array}{l}\text { Note de lésions cæcales } \\
\text { (survivants et morts) }\end{array}$ & (C) & 3,0 & 1,9 & 2,2 & 2,4 \\
\hline \multicolumn{2}{|l|}{$\begin{array}{l}\text { Excrétion d'ookystes } \\
\text { par animal : }\end{array}$} & & & & \\
\hline \multicolumn{2}{|l|}{$\begin{array}{l}\mathrm{J}+5 \\
\mathrm{~J}+6 \\
\mathrm{~J}+7\end{array}$} & $\begin{array}{r}1890 \\
450 \\
250\end{array}$ & $\begin{array}{r}1310 \\
320 \\
77\end{array}$ & $\begin{array}{r}1080 \\
910 \\
124\end{array}$ & $\begin{array}{l}730 \\
530 \\
107\end{array}$ \\
\hline \multicolumn{2}{|l|}{ Total } & 2590 & 1707 & 2114 & 1367 \\
\hline \multirow{2}{*}{\multicolumn{2}{|c|}{$\begin{array}{l}\text { Matières fécales (notes): } \\
\mathrm{J}+5 \\
\mathrm{~J}+6 \\
\mathrm{~J}+7\end{array}$}} & & & & \\
\hline & & $\begin{array}{l}4 \\
1 \\
1\end{array}$ & $\begin{array}{l}3 \\
2 \\
1\end{array}$ & $\begin{array}{l}2 \\
1 \\
0\end{array}$ & $\begin{array}{l}3 \\
2 \\
1\end{array}$ \\
\hline \multirow{2}{*}{$\begin{array}{l}\text { Consommation d'eau par jour } \\
\text { et par animal (g) }\end{array}$} & (T) & 47,5 & 42,3 & 43,6 & 46,3 \\
\hline & (C) & 47,5 & 44,4 & 43,5 & 45,5 \\
\hline \multirow{2}{*}{$\begin{array}{l}\text { Consommation d'aliment par } \\
\text { jour et par animal }(\mathrm{g})\end{array}$} & (T) & 30,9 & 27,3 & 29,8 & 31,0 \\
\hline & (C) & 27,0 & 26,1 & 27,7 & 28,3 \\
\hline \multirow[t]{2}{*}{ Indice de consommation } & (T) & 2,05 & 2,76 & 2,37 & 2,16 \\
\hline & (C) & 2,38 & 2,75 & 2,84 & 2,72 \\
\hline
\end{tabular}


TABleau 2

Analyse de variance du gain de poids et du poids à l'abattage.

Variance analysis for body weight gain and weight at slaughter time.

\begin{tabular}{|c|c|c|c|c|}
\hline \multirow[b]{2}{*}{ Source de variation } & \multirow{2}{*}{$\begin{array}{l}\text { Degrés } \\
\text { de liberté }\end{array}$} & \multicolumn{3}{|c|}{ Valeur de $F$ par caractère et signification } \\
\hline & & $\begin{array}{c}\text { Gain de poids } \\
(\Delta P) \\
\text { absorbé }\end{array}$ & $\begin{array}{c}\text { Poids } \\
\text { à l'abattage } \\
\left(\mathbf{P}^{\prime}\right)\end{array}$ & $\begin{array}{l}\text { Gain de poids } \\
\text { relatif }\end{array}$ \\
\hline Traitement & 1 & $73,17^{* * *}$ & $2,69 * * *$ & $50,91^{* * *}$ \\
\hline Type génétique. & 3 & $35,81^{* * *}$ & $101,37^{* * *}$ & 0,58 \\
\hline Interaction . . & 3 & $5,29 * * *$ & $4,33^{* *}$ & $4,01^{* *}$ \\
\hline Résiduelle & 209 & & & \\
\hline
\end{tabular}

** : significatif au seuil 1 p. cent.

*** : significatif au seuil 0,1 p. cent.

Le tableau 3 détaille la répartition des différents groupes, dans le lot contaminé, suivant leur note de lésions caecales et donne le résultat du test de $\chi^{2}$ correspondant.

\section{TABleaU 3}

Répartition des animaux par note de lésion cacale suivant le type génétique (lot contaminé).

Distribution of birds according to caecal lesion score for each genetic type (inoculated).

\begin{tabular}{|c|c|c|c|c|c|}
\hline \multirow{2}{*}{ Lignée ou croisement } & \multicolumn{4}{|c|}{ Note } & \multirow{2}{*}{ Total } \\
\hline & 1 & 2 & 3 & 4 & \\
\hline Fayoumi & 4 & 33 & 1 & 0 & 38 \\
\hline R.I.R. $\ldots \ldots$ & 0 & 7 & 5 & 7 & 19 \\
\hline Fayoumi × R.I.R. & 1 & 26 & 2 & 3 & 32 \\
\hline R.I.R. $\times$ Fayoumi & 0 & 18 & 4 & 4 & 26 \\
\hline
\end{tabular}

Test en regroupant les notes $1+2$ et $3+4: \chi_{(3)}^{2}=28.34^{* * *}$

Le tableau 4 montre, pour chaque type génétique, le gain de poids moyen correspondant à chaque note de lésion. 


\section{TABLEAU 4}

Gain de poids moyens suivant la note individuelle de lésions cacales dans chaque type génétique.

Mean body weight gain according to caecal lesion score within genetic type.

\begin{tabular}{c|c|c|c|c}
\hline \hline \multirow{2}{*}{ Note de lésion } & \multicolumn{4}{|c}{ Lignée ou croisement (1) } \\
\cline { 2 - 5 } & R.I.R. & Fayoumi & R.I.R. $\times$ Fayoumi $\left(^{*}\right)$ & Fayoumi $\times$ R.I.R. \\
\hline \multirow{2}{*}{1} & - & $82,5^{(4)}$ & - & $90,0^{(1)}$ \\
2 & $114,6^{(7)}$ & $84,5^{(33)}$ & $101,5^{(16)}$ & $95,0^{(26)}$ \\
3 & $77,6^{(5)}$ & $105,0^{(1)}$ & $85,0^{(4)}$ & $54,5^{(2)}$ \\
$(4)^{(2)}$ & $(79,6)^{(7)}$ & - & $(68,5)^{(4)}$ & $(48,7)^{(3)}$ \\
\hline
\end{tabular}

(1) Effectifs entre parenthèses.

(2) A deux exceptions près, les animaux notés 4 sont morts le cinquième jour, suivant l'inoculation.

\section{Discussion}

\section{A. Critères de poids et consommation alimentaire}

En ce qui concerne le poids corporel à 13 jours d'âge, c'est-à-dire avant inoculation, le tableau 1 montre, comme on s'y attendait, qu'il est très voisin, dans chaque type génétique, pour les deux lots. Par la suite, le gain de poids et le poids atteint à l'abattage sont sensiblement abaissés dans le lot contaminé par comparaison au lot témoin. Le tableau 2 confirme que l'effet "traitement" est hautement significatif. L'effet "type génétique ", sur les mêmes caractères, visible au tableau 1 , se révèle lui aussi hautement significatif au tableau 2. On retrouve le fait connu que la Fayoumi a une plus petite taille, à tous âges, que la race Rhode-Island. Par contre, l'effet "type génétique "n'est pas significatif, dans le présent échantillon, pour le rapport du gain de poids au poids initial. On note enfin que les croisements sont intermédiaires pour tous les caractères de croissance avec, apparemment, une supériorité par rapport à la moyenne des lignées parentales lorsque le père est Fayoumi.

Le point le plus intéressant est la réponse comparée des 4 types génétiques à l'inoculation d'Eimeria tenella: on voit au tableau 1 que chez la Fayoumi, le gain de poids de 13 à 23 jours n'est diminué que de $4 \mathrm{p}$. cent lorsqu'on passe du lot témoin au lot contaminé, alors que la diminution correspondante est respectivement de 25,22 et 19 p. cent pour la lignée Rhode-Island M.99 et les deux croisements. Le tableau 2 confirme cette différence de réponse en faisant apparaitre un effet d'interaction type génétique $\times$ traitement hautement significatif, tant pour le gain de poids en valeur absolue que pour le poids à l'abattage et pour le gain de poids relatif (rapporté au poids de départ).

Quant à la consommation d'aliment, et quoique nous n'ayons pas fait d'analyse statistique sur ce caractère, une tendance analogue est suggérée au tableau 1: la consommation journalière moyenne est abaissée pour tous les types génétiques par l'inoculation d'Eimeria tenella, mais cette diminution est, proportionnellement, la plus

(*) Deux individus de ce croisement comptés au tableau 3 mais non pesés (morts accidentelles). 
faible pour les poulets Fayoumi et la plus élevée pour la lignée R.I.R. : en pourcentage du lot témoin, elle représente respectivement 12,$6 ; 4,4 ; 7,0$ et 8,7 p. cent pour les lignées R.I.R., Fayoumi, les croisements R.I.R. $\times$ Fayoumi, et Fayoumi $\times$ R.I.R. Cette diminution est moindre, dans l'ensemble, que celle de la croissance pondérale : l'indice de consommation est détérioré pour la lignée $R . I . R$. et les deux croisements (augmentant respectivement de 16,$0 ; 19,8$ et 25,9 p. cent) mais pas chez la Fayoumi ( $-0,4$ p. cent). Enfin la consommation d'eau est sensiblement la même dans le lot contaminé et dans le lot témoin.

\section{B. Mortalité, lésions et autres critères}

Les pourcentages de mortalité dans le lot contaminé (tabl. 1) diffèrent significativement entre types génétiques et se classent de façon parallèle aux gains de poids : les pertes sont les plus élevées dans la lignée M.99, nulles pour la Fayoumi, intermédiaires dans les croisements, sans qu'on puisse déceler une différence entre ces derniers. Le $\chi^{2}$ correspondant, à 3 degrés de liberté, est égal à $13,46(\mathrm{p}<0,005)$.

La note moyenne des lésions caecales reproduit encore le même classement, la Fayoumi étant la moins atteinte et la R.I.R. la plus affectée. Le tableau 3, qui donne la répartition des notes de lésions dans chaque type génétique, montre que les génotypes diffèrent très significativement à cet égard $(\mathrm{p}<0,001)$.

Le tableau 1 suggère, du $5^{\mathrm{e}}$ au $7^{\mathrm{e}}$ jour après l'inoculation, une excrétion totale d'ookystes plus faible chez la Fayoumi et le croisement Fayoumi $\times$ R.I.R. que chez la R.I.R. et une valeur intermédiaire du croisement R.I.R. $\times$ Fayoumi. Les notes concernant les matières fécales, enfin, suggèrent surtout des hémorragies plus importantes dans la lignée R.I.R.

\section{Corrélations entre gain de poids et note de lésion caecale}

Intra-groupes, le tableau 4 montre que, dans l'ensemble, plus la note de lésion est élevée, plus le gain de poids est abaissé en moyenne, même en excluant les animaux de note 4 qui, presque tous, sont morts à 20 jours d'âge. Cette observation ne semble pas concorder avec les conclusions de JEFFERS et al. (1970) qui ne trouvent pas de corrélation nette entre les divers critères de résistance, mortalité, lésions et gain de poids.

\section{v. Conclusions}

Le principal résultat de notre étude est évidemment la plus grande résistance de la Fayoumi à Eimeria tenella, comparée à la lignée Rhode-Island M.99, qui paraît, quant à elle, présenter une sensibilité "moyenne " par comparaison à d'autres lignées originaires d'Europe ou des Etats-Unis. Bien que des ookystes soient excrétés, on n'observe, pour la première, aucune chute de poids significative par rapport au lot témoin, aucune mortalité, les lésions caecales sont moins importantes et l'indice de consommation n'est pas modifié. On peut penser qu'une sélection naturelle s'est exercée chez la Fayoumi par suite des conditions sanitaires difficiles auxquelles elle a pu être soumise dans un contexte d'élevage "traditionnel». De façon analogue, 
ReHMAN (1972) trouve une résistance relativement élevée à Eimeria tenella dans une race locale indienne. Plus généralement, des différences considérables de mortalité causée par Eimeria tenella ont été trouvées entre des populations non sélectionnées préalablement dans ce sens (Challey et al., 1968 ; JefFers et al., 1970) et la possibilité de sélectionner sur ce critère a été démontrée (par exemple RosenBERG, 1941 ; Palafox et al., 1945 ; Rosenberg et al., 1954 ; Champion, 1954 ; Klimes \& Orel, 1968).

Les croisements $F_{1}$ présentent les signes d'une coccidiose aiguë. Cependant, leur mortalité est plus faible que celle des animaux R.I.R. Dans le détail, suivant les critères, les poussins $F_{1}$ sont, soit à peu près intermédiaires, soit plus proches de la lignée R.I.R., soit encore (dans un cas) plus proches de la Fayoumi. Au premier cas se rattachent la baisse de consommation alimentaire, la mortalité et la gravité des lésions caecales, alors que les deux $\mathrm{F}_{1}$ se rapprochent plutôt de la R.I.R. pour la baisse du gain de poids et l'augmentation de l'indice de consommation.

Nos données paraissent cö̈ncider avec celles d'auteurs antérieurs (notamment Champion, 1954), en ce sens que, comme eux, nous ne trouvons pas d'effet d'hétérosis pour la survie en réponse à une infection par Eimeria tenella. Challey et al. (1968) et JEFFERs et al. (1970) trouvent en moyenne une légère supériorité des croisements $F_{1}$ sur la moyenne des lignées parentales, mais l'avantage apparaît très variable selon les lignées croisées.

Sur les mécanismes physiologiques possibles de la différence trouvée entre races pures et croisements dans nos résultats, les données présentes n'apportent pas d'élément. Parmi les facteurs pouvant être responsables de différences de résistance à une autre coccidie, Eimeria necatrix, Gross (1976) a mentionné les modalités de la réponse aux diverses agressions, évaluée par le taux de corticostérone plasmatique. Une comparaison à cet égard des races et croisements étudiés ici pourrait être envisagée.

Dans une comparaison entre diverses lignées de pondeuses, d'une part pour leur résistance à Eimeria tenella, d'autre part pour leur mortalité générale en conditions naturelles, Challey et al. (1968) trouvent une corrélation positive entre ces deux caractères. Relativement à la race Fayoumi, l'opinion selon laquelle elle possède une résistance à une variété d'agents pathogènes serait en accord avec les observations de ces auteurs.

Reçu pour publication en juin 1982

\section{Summary}

\section{Studies on the Fayoumi poultry breed}

II. - Resistance to coccidiosis (Eimeria tenella) of Fayoumi, Rhode-Island and F, chicken

Day-old chicks of the breeds Fayoumi, Rhode-Island and their two reciprocal crosse were distributed at random in a Control an a Treated group. In this latter group each individual was inoculated with 200.000 ookysts at 15 days of age. All chicks were slaughtered at 23 days. Mortality from inoculation to slaughter age, weight gain of survivors and a visual score of caecal lesions were recorded individually. Food and water intake were measured by groups. Daily oocyst excretion and a visual score of faeces were noted on a sample of each breed or cross. For all criteria, Fayoumi chicks appeared as the most resistant to Eimeria tenella. In particular, they had no mortality, their weight gain was depressed by only 4 per cent as compared to controls, and their average caecal lesion score was the least. In all these respects, Rhode-Island chicken were the most susceptible and the two crosses were intermediate. 


\section{Références bibliographiques}

Challey J.R.T., JefFers K., Mc GibBon W.H., 1968. The response of commercial egg production stocks to experimental infection with Eimeria tenella and its relationship to field mortality. Poult. Sci., 47, 1197-1204.

Champion L.R., 1954. The inheritance of resistance to cecal coccidiosis in the domestic fowl. Poult. Sci., 33, 670-681.

Gross W.B., 1976. Plasma steroid tendency, social environment and Eimeria necatrix infection. Poult. Sci., 55, 1508-1512.

Hamet N., Robin B., Toucas L., 1981. Etude de la variation de l'excrétion ookystale dans differents élevages de poulets de chair. Bull. d'Information, Station expérimentale d'Aviculture de Ploufragan, 21, 20-26.

Jeffers T.K., Challey J.R., MC GibBon W.H., 1970. Response of several lines of fowl and their single cross progeny to experimental infection with Eimeria tenella. Avian Dis., 14, 203-210.

JOHNSON Joyce, REID W.M., 1970. Anticoccidial drugs : lesion scoring technique in Battery and Floor Pen experiments with chickens. Expl. Parasit., 28, 30-36.

Khodali A.R., Shomein A.M., 1974. Studies of spirochetosis in fowls in the Sudan. I. Epizootiology and experimental transmission. Bull. Epizootic dis. of Africa, 22, 251-254.

KLIMES B., OREL V., 1969. Investigations of genetic resistance to coccidiosis. I. - Selection response of chicks to infection by Eimera tenella. Acta Vet. (Brno), 38, 51-57.

NoRdskog A.W., Philips P.E., 1960. Heterosis in poultry. V. Reciprocal crosses involving Leghorn, heavy breeds and Fayoumi. Poult. Sci., 39, 257-263.

Palafox A.L., Alicata J.E., Kartman L., 1949. Breeding chickens for resistance to cecal coccidiosis. Wld Poult. Sci. J., 5, 84-87.

PRINCE A.M., 1958. Quantitative studies on Rous Sarcoma virus. II. Mechanisms of resistance of chick embryos to chorio-allantoic inoculation of Rous Sarcoma virus. J. Nat. Cancer Inst., 20, 843-850.

ReHman B., 1972. Comparative studies on the immune response in Desi and foreign breeds of chickens against Eimeria tenella. Pakist. J. Sci., 23, 201-204.

RoSEnberg M.M., 1941. A study of the inheritance of resistance to Eimeria tenella in the domestic fowl. Poul. Sci., 20, 472 (abstr.).

Rosenberg M.M., Alicaza J.F., Palafox A.L., 1954. Further evidence of hereditary resistance to cecal coccidiosis in chickens. Poult. Sci., 33, 972-980. 